\title{
DESENVOLVIMENTO E VALIDAÇÃO DE MÉTODO ANALÍTICO POR CLAE PARA A QUANTIFICAÇÃO SIMULTÂNEA DE DIPROPIONATO DE BETAMETASONA E FOSFATO SÓDICO DE BETAMETASONA EM SUSPENSÃO INJETÁVEL
}

\author{
Alice Simon, Lúcio Mendes Cabral e Valeria Pereira de Sousa* \\ Departamento de Medicamentos, Faculdade de Farmácia, Universidade Federal do Rio de Janeiro, Av. Carlos Chagas Filho, 373 , \\ CCS, Bloco Bss, Cidade Universitária, 21944-970 Rio de Janeiro - RJ, Brasil \\ Recebido em 8/4/11; aceito em 9/9/11; publicado na web em 8/11/11
}

\begin{abstract}
DEVELOPMENT AND VALIDATION OF AN ANALYTICAL METHOD BY HPLC FOR SIMULTANEOUS QUANTIFICATION OF BETAMETHASONE DIPROPIONATE AND BETAMETHASONE SODIUM PHOSPHATE IN INJECTABLE SUSPENSION. A reversed-phase HPLC method was developed and validated to separate and simultaneously quantify the association of betamethasone sodium phosphate (BP) and betamethasone dipropionate (BD) in injectable suspensions. Chromatographic conditions were ternary gradient elution at $1.6 \mathrm{~mL} / \mathrm{min}$ on a C18 column with $254 \mathrm{~nm}$. The linearity of the method was established in the range 120 to 280 $\mathrm{mg} / \mathrm{mL} \mathrm{BD}$, and 48 to $112 \mathrm{mg} / \mathrm{mL}$ BP. The RSD of intermediate precision of the method was < $1 \%$ and recoveries were $99-101 \%$ for both drugs. The method proved selective, linear, precise, accurate and robust for quantifying BP and BD in commercial injectable suspensions.
\end{abstract}

Keywords: betamethasone; high performance liquid chromatography; injectable suspensions.

\section{INTRODUÇÃO}

Os glicocorticoides são importantes hormônios de estresse e, quando administrados em doses suprafisiológicas, exibem também efeitos anti-inflamatórios, antialérgicos e antirreumáticos terapeuticamente úteis. ${ }^{1}$ A betametasona é um corticosteroide, com atividade principal como glicocorticoide, suas propriedades mineralocorticoides são quase ausentes tornando-a adequada para o tratamento de condições em que a retenção de água seria uma desvantagem. ${ }^{2} \mathrm{~A}$ betametasona é comercializada como base livre e também como acetato, benzoato, dipropionato, fosfato sódico e valerato, em diferentes formas farmacêuticas. ${ }^{3}$

Uma importante forma farmacêutica contendo sais de betametasona é a suspensão aquosa injetável. Esta formulação é indicada para aplicação intramuscular, possuindo liberação imediata e sustentada, com tempo de meia vida plasmática de 3 a 5 h e tempo de meia vida tecidual de 36 a $54 \mathrm{~h}$. Segundo o fabricante do medicamento referência, o Diprospan ${ }^{\circledR}$ injetável é uma associação de ésteres de betametasona com efeito anti-inflamatório, antialérgico e antirreumático. A ação terapêutica imediata é fornecida pelo éster solúvel, fosfato sódico de betametasona (FB), presente na concentração de $2 \mathrm{mg} / \mathrm{mL}$, o qual é rapidamente absorvido após injeção. A ação prolongada é promovida pelo dipropionato de betametasona (DB), presente na concentração de $5 \mathrm{mg} / \mathrm{mL}$.

$\mathrm{O}$ DB $\left(\mathrm{C}_{28} \mathrm{H}_{37} \mathrm{FO}_{7}\right.$, massa molecular $\left.=504,6\right)$ é praticamente insolúvel em água, solubilidade limitada em álcool, livremente solúvel em acetona, diclorometano e em clorofórmio. $\mathrm{O} F B\left(\mathrm{C}_{22} \mathrm{H}_{28} \mathrm{FNa}_{2} \mathrm{O}_{8} \mathrm{P}\right.$, massa molécula $\mathrm{r}=516,4$ ) é solúvel em água, álcool di-hidratado, livremente solúvel em metanol, praticamente insolúvel em acetona, diclorometano, éter e em clorofórmio. ${ }^{2,4}$

Os métodos analíticos empregados no controle da qualidade rotineiro dos lotes de medicamentos produzidos numa indústria também apresentam importância considerável em estudos de equivalência farmacêutica. Neste tipo de estudo se avalia a qualidade do medicamento teste por meio de análise comparativa com o medicamento referência, tornando importante a disponibilidade de um método

*e-mail: valeria@pharma.ufrj.br oficial e validado para ser empregado no processo de registro de medicamentos genéricos e similares. ${ }^{5-8}$

Até o presente momento, constata-se ausência de monografia com a descrição de um método de quantificação para suspensão injetável destes fármacos na Farmacopeia Brasileira ${ }^{5}$ e nos principais compêndios oficiais reconhecidos pela Agência Nacional de Vigilância Sanitária segundo a Resolução n $37,{ }^{9}$ Farmacopeia Americana, ${ }^{10}$ Britânica, ${ }^{11}$ Mexicana, ${ }^{12}$ Francesa, ${ }^{13}$ Europeia,${ }^{14}$ Portuguesa, ${ }^{15}$ Internacional ${ }^{16} \mathrm{e}$ Japonesa ${ }^{17}$. Nas farmacopeias consultadas são descritos métodos por CLAE e espectrofotometria para a quantificação das matérias-primas isoladas. ${ }^{10,11,14,17}$ Atualmente esta suspensão injetável em estudo é produzida e comercializada no mercado brasileiro por cerca de vinte empresas farmacêuticas, tornando necessário um método analítico validado para o controle de qualidade e equivalência farmacêutica.

Portanto, este estudo descreve o desenvolvimento e a validação de um método por cromatografia líquida de alta eficiência com detecção por UV para a quantificação de FB e DB em suspensões injetáveis, de forma a auxiliar no controle de qualidade rotineiro dessa associação de fármacos.

\section{PARTE EXPERIMENTAL}

\section{Materiais}

Produtos farmacêuticos, substâncias químicas de referência e solventes

Os padrões de trabalho de DB e FB, medicamento referência e placebo foram doados pela Mantecorp (Rio de Janeiro, Brasil). O placebo contém cloreto de sódio, edetato dissódico, polissorbato 80, álcool benzílico, metilparabeno, propilparabeno, carboximetilcelulose sódica e propilenoglicol. Foi adquirida no mercado nacional suspensão injetável intramuscular da associação de DB e FB, de 10 fabricantes diferentes (medicamento referência, genéricos e similares), nomeados produtos A a J. Os solventes utilizados, acetonitrila $(\mathrm{ACN})$ e metanol, tinham grau cromatográfico e foram adquiridos da Tedia (Rio de Janeiro, Brasil) e o fosfato de potássio monobásico anidro da Vetec (Rio de Janeiro, Brasil). 


\section{Equipamentos}

Foi utilizado cromatógrafo da marca Elite LaChrom equipado com sistema de bomba quaternária L-2130, forno de coluna L-2300, autoinjetor L-2200 e detector DAD L-2455. Os dados cromatográficos foram analisados através do programa EZChrom Elite. Na avaliação da robustez foi utilizado um segundo cromatógrafo da marca Shimadzu 10A, equipado com sistema de bomba quaternária LC-10AD, autoinjetor SIL-10AD e detector DAD SPD-M10APDA. Os dados foram adquiridos com programa Class-VP 6.1. Foi utilizada na validação a coluna cromatográfica Kromasil 100-5 C18 (150 x $4,6 \mathrm{~mm} ; 5 \mu \mathrm{m})$.

\section{Método}

\section{Parâmetros cromatográficos}

A fase móvel foi constituída de gradiente de acetonitrila, metanol e tampão fosfato de potássio monobásico $0,07 \mathrm{M}$, descrito na Tabela 1 . A solução tampão foi preparada em água destilada, conforme descrito na Farmacopeia Americana. ${ }^{10} \mathrm{~A}$ fase móvel foi filtrada em membrana Millipore $0,45 \mu \mathrm{m}$ e degaseificada antes do uso. As análises foram efetuadas a uma temperatura de $25^{\circ} \mathrm{C}$ com fluxo da fase móvel de 1,6 $\mathrm{mL} / \mathrm{min}$ e o volume de injeção de $10 \mu \mathrm{L}$. As análises foram fixadas em 17 min de duração, empregando detecção no ultravioleta a $254 \mathrm{~nm}$.

\section{Preparo da solução estoque padrão}

Transferiram-se cerca de $50 \mathrm{mg}$ de padrão de DB e $20 \mathrm{mg}$ de padrão de FB para balão volumétrico de $100 \mathrm{~mL}$. Adicionaram-se $50 \mathrm{~mL}$ de acetonitrila e $30 \mathrm{~mL}$ de água. Após $15 \mathrm{~min}$ no ultrassom, ou até a completa solubilização, o volume foi completado com água, obtendo-se uma solução estoque padrão de 500 e $200 \mu \mathrm{g} / \mathrm{mL}$ dos fármacos, respectivamente.

\section{Preparo da solução amostra}

Transferiu-se o volume de uma ampola, equivalente a $5 \mathrm{mg}$ de $\mathrm{DB}$ e $2 \mathrm{mg}$ de $\mathrm{FB}$, para balão volumétrico de $25 \mathrm{~mL}$. Adicionaram-se $12,5 \mathrm{~mL}$ de acetonitrila e 7,5 $\mathrm{mL}$ de água. $\mathrm{O}$ mesmo procedimento adotado para o preparo da solução padrão foi seguido, obtendo-se uma solução de 200 e $80 \mu \mathrm{g} / \mathrm{mL}$ dos fármacos, respectivamente. As soluções foram filtradas em filtro Millipore de membrana PVDF de $0,45 \mu \mathrm{m}$ antes das injeções.

\section{Validação do método analítico}

Os ensaios para a validação do método proposto foram realizados conforme as recomendações contidas na legislação vigente, a resolução RE 899 da Agência Nacional de Vigilância Sanitária (ANVISA) ${ }^{7}$ e no Guia do Internacional Conference on Harmonisation (ICH) ${ }^{8}$ os quais descrevem que testes de quantificação para a determinação do princípio ativo em produtos farmacêuticos devem apresentar especificidade, linearidade, precisão, limite de quantificação, limite de detecção, exatidão e robustez adequados à análise pretendida. ${ }^{7,8} \mathrm{Na}$ validação do método analítico foi utilizado lote piloto do medicamento referência e seu placebo, doados pela empresa produtora.

A especificidade do método foi determinada em cromatógrafo acoplado a detector de arranjo de fotodiodos (DAD) e analisada através do programa EZChrom Elite. Para isso, foram injetadas soluções padrão de DB e FB, da forma farmacêutica (suspensão injetável) e do placebo nas concentrações de 200 e $80 \mu \mathrm{g} / \mathrm{mL}$, respectivamente. Para verificar a similaridade foram realizadas varreduras espectrais no início, meio e fim dos picos e os espectros do padrão e da amostra foram comparados e o índice de similaridade avaliado. Determinouse o índice de pureza dos picos através de ratiogram, o qual define a razão da relação das absorbâncias em função do tempo de eluição.
Para procedimentos cromatográficos, cromatogramas representativos devem ser utilizados para demonstrar a especificidade. ${ }^{8}$

A linearidade do método foi verificada a partir de três curvas com cinco níveis de concentrações. A solução estoque padrão foi diluída com acetonitrila e água (50:50; v/v), a fim de se obter concentrações aproximadas de 120,160, 200, 240 e $280 \mu \mathrm{g} / \mathrm{mL}$ de DB, e concentrações aproximadas de 48, 64, 80, 96 e $112 \mu \mathrm{g} / \mathrm{mL}$ de FB. Todas as soluções finais foram filtradas em filtro Millipore $0,45 \mu \mathrm{m}$ antes das injeções. Os resultados obtidos foram tratados estatisticamente através de análise de regressão linear pelo método dos mínimos quadrados. O critério mínimo aceitável do coeficiente de correlação foi de $0,99{ }^{7,8}$

A precisão foi avaliada em dois níveis, repetibilidade (intradia) e precisão intermediária (interdias) em dias consecutivos, para soluções nas concentrações de 80,100 e $120 \%$ com três réplicas cada. As soluções foram analisadas e, a seguir, o desvio padrão relativo (DPR) foi calculado, valores acima de $5 \%$ não foram admitidos. ${ }^{7,8}$

Os limites de quantificação (LQ) e de detecção (LD) foram estimados com base no desvio padrão da resposta e da inclinação da curva de calibração dos analitos através das equações LQ = DP x 10/IC e $\mathrm{LD}=\mathrm{DP} \times 3 / \mathrm{IC}$, nas quais DP é o desvio padrão do intercepto com o eixo y de três curvas de calibração e IC é a média dos coeficientes angulares das respectivas curvas. ${ }^{7,8}$

A exatidão foi calculada como porcentagem de recuperação, através da relação entre a concentração média determinada experimentalmente e a concentração teórica correspondente. ${ }^{7,8} \mathrm{O}$ percentual recuperado deve estar entre 98 e $102 \%$ do valor verdadeiro. ${ }^{8} \mathrm{O}$ estudo da exatidão foi realizado pela adição de padrão à solução de placebo do medicamento referência nas concentrações de 80, 100 e $120 \%$ com três réplicas cada. As soluções foram analisadas e, posteriormente, calculou-se o percentual de recuperação.

A robustez do método analítico foi avaliada através da análise do teor dos fármacos no medicamento referência em diferentes condições cromatográficas, produzidas por alterações no $\mathrm{pH}$ da fase móvel $(4,01 ; 4,45 ; 4,97)$, temperatura $\left(25-30{ }^{\circ} \mathrm{C}\right)$, fluxo $(1,5-1,6 \mathrm{~mL} /$ min) e mudança de equipamento (Elite LaChrom - Shimadzu 10A).

\section{Doseamento da suspensão injetável de $D B$ e FB}

O doseamento da suspensão injetável foi realizado utilizando dez diferentes produtos disponíveis no mercado, incluindo medicamento referência, genéricos e similares, nomeados de A a J. As amostras foram preparadas conforme descrito em Preparo da solução amostra. Foi determinada a equação da reta pela concentração versus área para cada fármaco, através de cinco diferentes concentrações do padrão. O teor dos fármacos foi calculado utilizando a equação da reta dos fármacos obtida por regressão linear.

\section{Análise estatística}

Os dados foram analisados estatisticamente utilizando-se análise de regressão linear e análise de variância (ANOVA) através do programa GraphPad Prism, com nível de significância de $\alpha<0,05$.

\section{RESULTADOS E DISCUSSÃO}

É fundamental que um método de quantificação por CLAE empregado para análise de produtos farmacêuticos de diferentes procedências seja seletivo, pois as formulações comerciais normalmente apresentam diferentes características e constituintes.

Após extensa busca bibliográfica foi encontrado um artigo que relata o desenvolvimento e validação de um ensaio de quantificação simultâneo da associação de DB e FB, porém ao aplicarmos a metodologia a separação não foi adequada. ${ }^{18}$ Desta forma, como ponto de partida para o desenvolvimento do método de quantificação da associação de DB e FB numa única corrida, foram utilizadas as variadas 
Tabela 1. Condições cromatográficas utilizadas no desenvolvimento do método e parâmetros cromatográficos obtidos

\begin{tabular}{|c|c|c|c|c|c|c|c|c|c|c|c|c|}
\hline & \multicolumn{5}{|c|}{ Condições cromatográficas } & \multicolumn{7}{|c|}{ Parâmetros cromatográficos } \\
\hline & \multirow{2}{*}{$\mathrm{t}(\min )$} & \multirow{2}{*}{$\mathrm{ACN} \%$} & \multirow{2}{*}{$\mathrm{TP} \%$} & \multirow{2}{*}{$\mathrm{F}$} & & \multicolumn{3}{|c|}{ FB } & \multicolumn{3}{|c|}{ DB } & \multirow{2}{*}{$\mathrm{R}_{\mathrm{s}}$} \\
\hline & & & & & & $t_{R}$ & $\mathrm{~A}$ & PT & $t_{R}$ & $\mathrm{~A}$ & PT & \\
\hline 1 & $\begin{array}{c}0-13 \\
13-30\end{array}$ & $\begin{array}{l}30 \\
50\end{array}$ & $\begin{array}{l}70 \\
50\end{array}$ & \multicolumn{2}{|l|}{1,5} & 3,50 & 0,89 & 623 & 23,37 & 1,22 & 30393 & 28,36 \\
\hline 2 & $\begin{array}{c}0-10 \\
10-30\end{array}$ & $\begin{array}{l}25 \\
50\end{array}$ & $\begin{array}{l}75 \\
50\end{array}$ & \multicolumn{2}{|l|}{1,5} & 6,40 & 0,72 & 983 & 23,92 & 1,22 & 31030 & 25,79 \\
\hline 3 & $\begin{array}{c}0-10 \\
12-22\end{array}$ & $\begin{array}{l}25 \\
60\end{array}$ & $\begin{array}{l}75 \\
40\end{array}$ & \multicolumn{2}{|l|}{1,5} & 6,57 & 1,54 & 4051 & 18,85 & 1,18 & 52984 & 33,18 \\
\hline 4 & $\begin{array}{c}0-6 \\
6-13 \\
13-17\end{array}$ & $\begin{array}{l}25 \\
60 \\
25\end{array}$ & $\begin{array}{l}75 \\
40 \\
75\end{array}$ & 1,5 & & 6,76 & 1,94 & 5732 & 13,72 & 1,50 & 34085 & 21,26 \\
\hline 5 & $\begin{array}{c}0-7 \\
8-20 \\
\end{array}$ & $\begin{array}{l}40 \\
60 \\
\end{array}$ & $\begin{array}{l}60 \\
40 \\
\end{array}$ & 1,2 & & 2,52 & 1,25 & 340 & 13,01 & 0,58 & 2779 & 13,67 \\
\hline & $\mathrm{t}(\min )$ & $\mathrm{ACN} \%$ & AA $\%$ & $\mathrm{~F}$ & & & $\begin{array}{c}\mathrm{FB} \\
\mathrm{A}\end{array}$ & $\mathrm{PT}$ & $t_{\mathrm{b}}$ & $\frac{\mathrm{DB}}{\mathrm{A}}$ & PT & $\mathrm{R}_{\mathrm{s}}$ \\
\hline 6 & $\begin{array}{c}0-6 \\
13-17\end{array}$ & $\begin{array}{l}20 \\
70\end{array}$ & $\begin{array}{l}80 \\
30\end{array}$ & 1,2 & & 9,97 & 1,07 & 600 & 15,87 & 0,61 & 25249 & 5,82 \\
\hline 7 & $\begin{array}{c}0-10 \\
15-17\end{array}$ & $\begin{array}{l}40 \\
60\end{array}$ & $\begin{array}{l}60 \\
40\end{array}$ & 1,2 & & -- & -- & -- & 11,83 & 0,60 & 3755 & -- \\
\hline 8 & $\begin{array}{c}0-3 \\
7-12 \\
15-20 \\
\end{array}$ & $\begin{array}{l}20 \\
75 \\
20 \\
\end{array}$ & $\begin{array}{l}80 \\
25 \\
80 \\
\end{array}$ & 1,2 & & 6,51 & 1,55 & 886 & 10,10 & 0,79 & 18596 & 6,12 \\
\hline & $\mathrm{t}(\min )$ & МET\% & $\mathrm{AA} \%$ & $\mathrm{~F}$ & & & FB & & & DB & & $\mathrm{R}_{\mathrm{s}}$ \\
\hline & (1) & & & & & $t_{R}$ & A & PT & $t_{R}$ & A & PT & \\
\hline 9 & $\begin{array}{c}0-2 \\
6-12 \\
14-17\end{array}$ & $\begin{array}{l}30 \\
80 \\
30\end{array}$ & $\begin{array}{l}70 \\
20 \\
70\end{array}$ & 1,2 & & 7,61 & 1,90 & 338 & 10,68 & 0,75 & 6497 & 2,80 \\
\hline 10 & $0-17$ & $\begin{array}{l}60 \\
80\end{array}$ & $\begin{array}{l}40 \\
20 \\
\end{array}$ & 1,2 & & -- & -- & -- & 13,58 & 0,65 & 3037 & -- \\
\hline & $\mathrm{t}(\min )$ & МET\% & $\mathrm{TP}$ & $\mathrm{F}$ & & & FB & & & DB & & $\mathrm{R}_{\mathrm{s}}$ \\
\hline & (न) & 1015 1 - & 11 & 1 & & $t_{R}$ & A & PT & $t_{R}$ & A & PT & $N_{s}$ \\
\hline 11 & $\begin{array}{c}0-8 \\
9-25\end{array}$ & $\begin{array}{l}58 \\
75\end{array}$ & $\begin{array}{l}42 \\
25\end{array}$ & 1,2 & & 5,99 & 0,68 & 436 & 15,54 & 1,31 & 36561 & 20,15 \\
\hline 12 & $\begin{array}{l}0-8 \\
9-17\end{array}$ & $\begin{array}{l}45 \\
80\end{array}$ & $\begin{array}{l}55 \\
20\end{array}$ & 1,2 & & 10,9 & 0,79 & 2684 & 14,07 & 1,59 & 60322 & 13,63 \\
\hline 13 & $\begin{array}{c}0-8 \\
9-17 \\
\end{array}$ & $\begin{array}{l}50 \\
80 \\
\end{array}$ & $\begin{array}{l}50 \\
20 \\
\end{array}$ & 1,2 & & 6,24 & 0,72 & 1078 & 13,81 & 1,41 & 59569 & 15,33 \\
\hline & $\mathrm{t}(\min )$ & MET\% & $\mathrm{ACN} \%$ & ТP \% & $\mathrm{F}$ & & FB & & & DB & & $\mathrm{R}_{\mathrm{s}}$ \\
\hline & (IIIII) & IVIL 180 & ACIV & 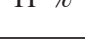 & 1 & $t_{R}$ & $\mathrm{~A}$ & PT & $t_{R}$ & $\mathrm{~A}$ & PT & $\mathrm{N}_{\mathrm{s}}$ \\
\hline 14 & $\begin{array}{c}0-6 \\
7-13 \\
14-17\end{array}$ & $\begin{array}{c}55 \\
-- \\
55 \\
\end{array}$ & $\begin{array}{l}-- \\
60 \\
--\end{array}$ & $\begin{array}{l}55 \\
40 \\
55\end{array}$ & 1,2 & 6,30 & 0,92 & 1728 & 13,53 & 1,58 & 41504 & 16,59 \\
\hline 15 & $\begin{array}{c}0-6 \\
7-13 \\
14-17\end{array}$ & $\begin{array}{l}60 \\
-- \\
60\end{array}$ & $\begin{array}{l}-- \\
60 \\
--\end{array}$ & $\begin{array}{l}40 \\
40 \\
40\end{array}$ & 1,2 & 4,10 & 1,0 & 1349 & 13,25 & 1,55 & 37827 & 25,47 \\
\hline 16 & $\begin{array}{c}0-6 \\
7-13 \\
14-17 \\
\end{array}$ & $\begin{array}{c}50 \\
-- \\
50 \\
\end{array}$ & $\begin{array}{l}-- \\
60 \\
-- \\
\end{array}$ & $\begin{array}{l}50 \\
40 \\
50 \\
\end{array}$ & 1,6 & 6,93 & 1,10 & 4648 & 12,95 & 1,02 & 64886 & 19,73 \\
\hline
\end{tabular}

t - tempo de corrida; ACN - acetonitrila; MET - metanol; TP - tampão fosfato de potássio 0,07M; AA - água acidificada; F - fluxo da fase móvel (mL/min); $\mathrm{t}_{\mathrm{R}}$ - tempo de retenção; $\mathrm{A}$ - assimetria; $\mathrm{PT}$ - pratos teóricos; $\mathrm{R}$ - resolução; $\mathrm{N}$ - eficiência.

condições cromatográficas descritas na Farmacopeia Americana ${ }^{10}$ para o doseamento dos fármacos como matéria-prima e quando presentes isoladamente em outras formulações.

Foram testadas condições utilizando de 30 a $70 \%$ de ACN com água ou tampão em modo isocrático e, também, diversas proporções de ACN na faixa de 10 a $75 \%$ com água em modo gradiente, com diferentes programações de tempo. Nestas condições observou-se que o pico do FB elui no volume morto e o pico do DB apresenta parâmetros cromatográficos inadequados em um tempo de retenção elevado. Testou-se um total de 14 gradientes em sistema binário utilizando ACN e tampão em diferentes proporções, tempo e fluxo (1,0 a 1,6 mL/min). Alguns dos gradientes promoveram a separação dos fármacos, porém em nenhum dos casos se obteve parâmetros cromatográficos satisfatórios. A Tabela 1 apresenta algumas das condições testadas e os parâmetros cromatográficos obtidos. Pode ser observado na condição 3 da Tabela 1 que o gradiente que utilizou uma programação de 25 a $60 \%$ de ACN com fluxo de $1,5 \mathrm{~mL} / \mathrm{min}$ resultou na eluição dos dois picos em tempos de corrida satisfatórios, porém com picos sem adequada simetria e pureza. Alterações no gradiente não foram eficientes para se obter uma separação adequada, como 
pode ser visto nas condições 1, 2, 4 e 5 da Tabela 1 . Foram avaliados gradientes em sistema binário utilizando $\mathrm{ACN}$ e água acidificada em diferentes proporções, tempo e fluxo a fim de melhorar os parâmetros cromatográficos, porém não foram obtidas melhoras significativas (Tabela 1, condições 6 a 8).

Novos testes foram realizados alterando a fase orgânica para metanol. Numa proporção de $50 \%$ de metanol com água ou tampão em modo isocrático, o FB em ambos os casos elui próximo ao volume morto e o pico do DB não é observado. Em gradientes com diferentes fluxos e proporções de 45 a $80 \%$ de metanol com tampão, observouse a eluição de picos sem simetria e resolução adequadas, conforme pode ser visto nas condições 11 a 13 da Tabela 1 . A utilização de um gradiente de 58 a $75 \%$ de metanol com tampão fosfato havia sido anteriormente descrita para essa separação, porém essa condição levou a um longo tempo de corrida com parâmetros cromatográficos inadequados (Tabela 1, condição 11). ${ }^{18}$ Testes utilizando água acidificada num sistema binário isocrático e em gradiente (Tabela 1, condições 9-10) com metanol apresentaram resultados insatisfatórios.

Foram testados sistemas ternários contendo ACN, metanol e tampão em diferentes proporções e fluxos (Tabela 1, condições 14 a 16). A condição 16 (Tabela 1), utilizando fluxo de 1,6 mL/min, apresentou parâmetros cromatográficos mais adequados para esta separação. A utilização de tampão $\mathrm{pH}$ 4,5 levou a uma melhor assimetria aos picos dos fármacos, provavelmente pela supressão iônica, por se tratarem de ácidos fracos. Outros artigos também demonstraram a necessidade de se utilizar sistema acidificado para obtenção de melhores separações cromatográficas da betametasona e seus sais. ${ }^{19-23}$ A condição 16 foi testada em coluna cromatográfica octilsilano, porém não foram observados valores adequados para os parâmetros cromatográficos. Desta forma, optou-se por manter a utilização de coluna cromatográfica ligada quimicamente a grupos octadecilsilano ( $150 \times$ 4,6 mm; $5 \mu \mathrm{m})$. Esta fase estacionária é descrita como a mais adequada para análises envolvendo a betametasona e seus sais. ${ }^{19-25}$

A fase móvel em sistema ternário de gradiente representada na condição 16, Tabela 1 , foi a mais adequada para a separação dos fármacos e dos constituintes da formulação. A maior sensibilidade analítica para os dois fármacos foi observada em comprimento de onda de $254 \mathrm{~nm}$, sendo também este o comprimento de onda sugerido pela Farmacopeia Americana nos métodos de quantificação de ambos os fármacos nas monografias das matérias-primas. ${ }^{10}$ Foi observado um alto valor de resolução para os picos dos fármacos, com tempos de retenção de 6,9 min para o FB e de 12,9 min para o DB, condição esta que não foi possível ser alterada pela presença de vários interferentes do placebo nos tempos 1,4; 2,4; 3,2 e 9,0 min. No processo de otimização do gradiente da fase móvel, a proporção inicial de metanol foi aumentada até $60 \%$ na tentativa de reduzir o tempo de retenção do FB. Estas condições levaram à assimetria do pico do DB. Em nenhuma das condições testadas foi possível aumentar a eficiência na separação do FB; este fato pode ser em parte explicado pela sua baixa concentração no injetável $(60 \%$ menor que o DB). A programação do gradiente 16 da Tabela 1 forneceu segurança na separação dos fármacos na presença dos diferentes excipientes presentes nos produtos comerciais, pois o pico está distante do tempo morto, onde elui a maior parte dos excipientes. Outros artigos utilizando a mesma fase estacionária relatam tempos de análises ainda maiores, como na separação do FB e acetato de betametasona no qual a separação é feita com 60 min de análise, utilizando um gradiente de dioxano e tetra-hidrofurano como fase orgânica. ${ }^{22} \mathrm{Fu}$ e colaboradores ${ }^{23}$ desenvolveram e validaram um método para a separação da betametasona base e suas substâncias relacionadas num gradiente utilizando terc-butanol e dioxano como fase orgânica e água acidificada como fase aquosa, com corridas de $68 \mathrm{~min}$. Shou e colaboradores ${ }^{21}$ descreveram um método utilizando solução acidificada e acetonitrila em corridas de 38 min para a separação de ácido salicílico, DB e suas substâncias relacionadas.

A Figura 1 apresenta os cromatogramas obtidos com o placebo (A), os padrões associados (B) e isolados (C e D) e o medicamento referência (E), diluídos em $\mathrm{ACN}$ e água (50:50; v/v) utilizando como fase móvel o gradiente 16 , descrito na Tabela 1 , coluna $C_{18}(150$ x 4,6 $\mathrm{mm} ; 5 \mu \mathrm{m}$ ), fluxo de $1,6 \mathrm{~mL} / \mathrm{min}$, detecção em $254 \mathrm{~nm}$, temperatura de $25^{\circ} \mathrm{C}$ e volume de injeção de $10 \mu \mathrm{L}$. No cromatograma $\mathrm{B}$, o pico do FB é observado no tempo de 6,93 min e apresentou pratos teóricos de 4660 e assimetria de 1,10 . O pico do DB, observado no tempo de 12,94 min, apresentou pratos teóricos de 64517 e assimetria igual a 1,01. O valor de resolução de 19,74 foi superior a 2,0, demonstrando a completa separação entre os fármacos mesmo na presença dos excipientes da formulação, sugerindo a seletividade do método proposto neste trabalho.

A identificação de cada fármaco no cromatograma da amostra foi possível pela comparação do tempo de retenção obtido no cromatograma dos respectivos padrões analisados isoladamente. A confirmação da identificação foi estabelecida pela comparação do espectro de absorbância no UV dos picos dos fármacos no cromatograma da amostra com seus respectivos padrões; neste caso, o programa indicou um alto índice de similaridade, variando entre $1 \mathrm{e}$ 0,9999 , entre a solução do padrão e a solução amostra, para ambos os fármacos. Os parâmetros cromatográficos calculados para os picos foram considerados satisfatórios, demonstrando que as condições cromatográficas são adequadas para a quantificação dos fármacos na suspensão injetável. O método cromatográfico proposto difere dos métodos descritos na literatura para os sais da betametasona. ${ }^{18-25}$

\section{Validação do método analítico}

A análise de pureza de pico é uma técnica de avaliação para detectar a presença de impurezas coeluídas com o pico do analito. ${ }^{26} \mathrm{O}$ método descrito neste trabalho foi considerado específico, como visto na Figura 2. A análise empregando detector DAD permitiu que fossem traçados os espectros no UV do pico dos fármacos em três tempos diferentes. Ambos os fármacos apresentaram espectros sobrepostos nos diferentes tempos em que foram analisados, FB (6,84; 6,94; 7,04 $\min )$ e DB $(12,92 ; 13,00 ; 13,08 \mathrm{~min})$, demonstrando que não apresentam diferenças significativas nos diferentes tempos, comprovando a ausência de substâncias interferentes e a especificidade do método. Com o auxílio do programa Elite LaChrom a razão da relação das absorbâncias em função do tempo de eluição gerou um ratiogram para cada pico, indicando que o índice de pureza dos picos é de 1,000 para ambos os fármacos, demonstrando que os picos cromatográficos do FB e do DB são atribuídos a um só componente. Na Figura 1 estão apresentados cromatogramas representativos da solução do placebo do injetável referência (A), dos padrões dos fármacos (B) e do medicamento referência (E). Pode-se notar que os fármacos apresentam separação adequada dos interferentes do placebo.

A Tabela 2 apresenta os resultados obtidos na análise de linearidade. A análise de regressão linear pelo método dos mínimos quadrados para as curvas dos fármacos DB e FB apresentou coeficientes de correlação acima de 0,999 , evidenciando uma alta correlação linear dentro dos limites das concentrações estudadas. Através da análise de variância pelo teste $\mathrm{F}$ foi analisada a validade da regressão e o modelo linear da curva analítica, comparando os valores de Fcalc $(\mathrm{F}$ calculado) e Ftab (F tabelado).

A validade da regressão é observada quando a inclinação da curva for significativamente diferente de zero, que é representada por valores de Fcalc superiores ao de Ftab. Os valores calculados para F foram Fcal $=51473,3$ para o FB e Fcalc $=72063,3$ para o DB, logo são superiores ao valor de Ftab de 6,41 (Fcalc >> Ftab), assim admite-se 

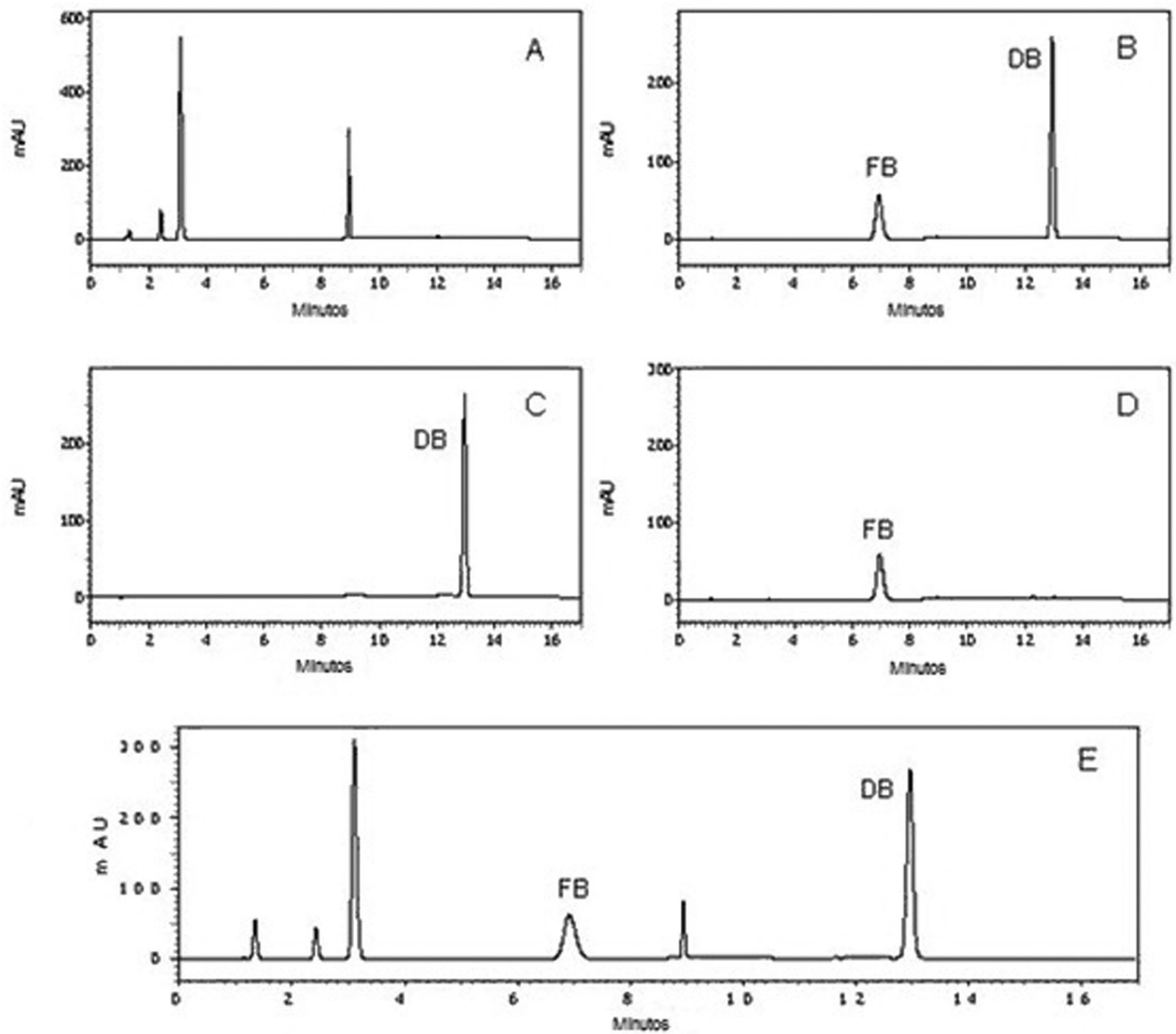

Figura 1. Cromatograma do placebo (A), solução do padrão dos fármacos $(B)$, solução do $F B(C)$, solução do $D B(D)$ e solução amostra - medicamento referência (E). Solução padrão de FB $80 \mu \mathrm{g} / \mathrm{mL}$ (6,95 min) e DB $200 \mu \mathrm{g} / \mathrm{mL}(12,95 \mathrm{~min}$ )

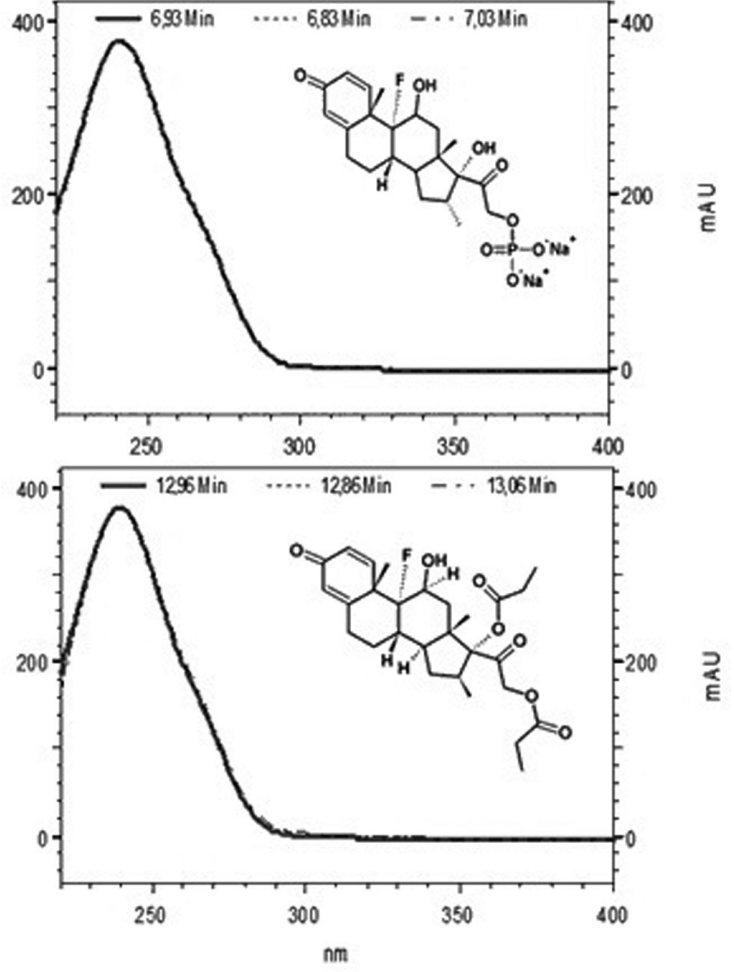

Figura 2. Pureza cromatográfica obtida por similaridade de espectros em diferentes tempos de retenção que a inclinação da reta não é nula e o ajuste linear da curva é aceito para ambos os fármacos com $\mathrm{P}<0,0001$. Os resultados apresentados na Tabela 2 demonstram que o Fcalc foi menor que o Ftab para a inclinação e o intercepto de ambos os fármacos; desta maneira, podese afirmar com $95 \%$ de confiança que o modelo é linear e está bem ajustado na faixa de concentração estudada. ${ }^{27}$

A partir das curvas de calibração dos fármacos foram estimados os limites de detecção e os limites de quantificação. Os resultados encontrados para o $\mathrm{DB}$ foram de $\mathrm{LD}=1,96 \mu \mathrm{g} / \mathrm{mL}$ e $\mathrm{LQ}=6,53 \mu \mathrm{g} /$ $\mathrm{mL}$, e para o $\mathrm{FB}$ foram de $\mathrm{LD}=0,14 \mu \mathrm{g} / \mathrm{mL}$ e $\mathrm{LQ}=0,48 \mu \mathrm{g} / \mathrm{mL}$. Considerando a concentração de trabalho de $200 \mu \mathrm{g} / \mathrm{mL}$ para o DB e de $80 \mu \mathrm{g} / \mathrm{mL}$ para o $\mathrm{FB}$, os valores encontrados para os limites de detecção e quantificação demonstram que o método tem sensibilidade adequada para a análise proposta.

Estão apresentados na Tabela 3 os valores obtidos na quantificação dos fármacos FB e DB na formulação injetável preparada nos níveis de 80,100 e $120 \%$ da concentração de trabalho, utilizando três replicatas para cada nível. O maior DPR encontrado na avaliação da precisão intermediária foi de $0,83 \%$ para o $\mathrm{FB}$ e de $0,82 \%$ para o DB. Estes valores estão bem abaixo do limite de $5 \%$ estipulado como adequado, indicando adequada repetibilidade (intradia) e precisão intermediária (interdias). ${ }^{7} \mathrm{Na}$ Tabela 3 podem ser observados valores fora do especificado para o DB $(120,6 \pm 0,67 \%)$. Isto se deve à utilização de lote piloto na validação do método. Foi testado um lote do medicamento referência adquirido no mercado local e os teores encontrados foram $100,14 \pm 5,82 \%$ para o DB e 97,07 $\pm 4,44 \%$ para o FB (média \pm DPR, $n=6$ ).

Outro parâmetro analítico avaliado, a exatidão, foi calculado 
Tabela 2. Análise estatística do estudo da linearidade

\begin{tabular}{|c|c|c|c|c|}
\hline Fármacos & Intervalo & Parâmetros & Resultados & $\begin{array}{c}\text { Estatística } \\
\alpha<0,05\end{array}$ \\
\hline \multirow{4}{*}{ Fosfato de betametasona } & \multirow{4}{*}{$40-120 \mu \mathrm{g} / \mathrm{mL}$} & Inclinação & $46580435,3 \pm 142716,1$ & $\begin{array}{r}\text { Fcalc }=2,61 \\
\text { Ftab }=4,06\end{array}$ \\
\hline & & Intercepto & $-112304,5 \pm 2244,7$ & $\begin{aligned} \text { Fcalc } & =1,51 \\
\text { Ftab } & =4,04\end{aligned}$ \\
\hline & & $\mathrm{R}$ & \multicolumn{2}{|c|}{$0,9999 \pm 1,10 \mathrm{E}-04$} \\
\hline & & Regressão & $\begin{array}{c}\text { Fcalc }=72063,3 \\
\text { Ftab }=6,41\end{array}$ & $P<0,0001$ \\
\hline \multirow{4}{*}{ Dipropionato de betametasona } & \multirow{4}{*}{$100-300 \mu \mathrm{g} / \mathrm{mL}$} & Inclinação & $39171243,4 \pm 62782,7$ & $\begin{aligned} \text { Fcalc } & =0,59 \\
\text { Ftab } & =4,06\end{aligned}$ \\
\hline & & Intercepto & $-116367,8 \pm 25588,7$ & $\begin{aligned} \text { Fcalc } & =0,89 \\
\text { Ftab } & =4,04\end{aligned}$ \\
\hline & & $\mathrm{R}$ & \multicolumn{2}{|c|}{$0,9999 \pm 3,72 \mathrm{E}-05$} \\
\hline & & Regressão & $\begin{array}{c}\text { Fcalc }=51473,3 \\
\text { Ftab }=6,41\end{array}$ & $P<0,0001$ \\
\hline
\end{tabular}

R - Coeficiente de correlação; Fcalc - F calculado; Ftab - F tabelado.

Tabela 3. Resultados obtidos na análise da repetibilidade e precisão intermediária

\begin{tabular}{|c|c|c|c|c|c|c|c|}
\hline \multirow{2}{*}{ Concentração (\%) } & \multirow{2}{*}{ Dia } & \multicolumn{3}{|c|}{ Fosfato sódico de betametasona } & \multicolumn{3}{|c|}{ Dipropionato de betametasona } \\
\hline & & Teor $(\%)$ & Intradia (DPR\%) & Interdia (DPR\%) & Teor $(\%)$ & Intradia (DPR\%) & Interdia (DPR\%) \\
\hline \multirow[t]{6}{*}{80} & 1 & 97,00 & 0,35 & 0,59 & 120,47 & 0,31 & 0,82 \\
\hline & & 97,34 & & & 120,12 & & \\
\hline & & 96,66 & & & 119,74 & & \\
\hline & 2 & 96,22 & 0,86 & & 119,91 & 1,21 & \\
\hline & & 96,74 & & & 119,64 & & \\
\hline & & 97,86 & & & 122,30 & & \\
\hline \multirow[t]{6}{*}{100} & 1 & 96,67 & 0,21 & 0,62 & 120,68 & 0,15 & 0,46 \\
\hline & & 96,56 & & & 120,33 & & \\
\hline & & 96,27 & & & 120,56 & & \\
\hline & 2 & 96,84 & 0,55 & & 120,91 & 0,36 & \\
\hline & & 97,91 & & & 121,79 & & \\
\hline & & 97,36 & & & 121,43 & & \\
\hline \multirow[t]{6}{*}{120} & 1 & 95,85 & 1,24 & 0,83 & 119,95 & 1,00 & 0,71 \\
\hline & & 95,24 & & & 119,33 & & \\
\hline & & 97,54 & & & 121,66 & & \\
\hline & 2 & 96,58 & 0,39 & & 120,99 & 0,41 & \\
\hline & & 96,60 & & & 120,95 & & \\
\hline & & 95,93 & & & 120,11 & & \\
\hline
\end{tabular}

através da porcentagem de recuperação da quantidade conhecida do analito adicionado ao placebo do medicamento. $\mathrm{O}$ critério de aceitação para a recuperação obtida é de $\pm 2 \%$, ou seja, entre 98,0 e $102,0 \%{ }^{8}$ Conforme observado na Tabela 4 , a porcentagem de recuperação dos fármacos ficou entre 99 e 101\%, dentro do critério de aceitação, demonstrando que o método é capaz de medir com exatidão os fármacos FB e DB na presença de outros componentes da suspensão injetável.

No estudo da robustez do método foram calculados os teores dos fármacos na suspensão injetável a cada alteração de parâmetro cromatográfico (Tabela 5). O teor médio (\%) e o DPR para o FB foi de $99,8 \pm 0,73$ e para o DB, $119,3 \pm 1,03$. Os baixos valores de DPR $(<5 \%)$ obtidos com a utilização de diferentes condições cromatográficas demonstram a robustez do método. O teor do DB está fora de especificação, como já explicado anteriormente, pois se trata de lote pi-
Tabela 4. Recuperação dos fármacos pelo método de CLAE

\begin{tabular}{lcc}
\hline Fármaco & Nível $(\mu \mathrm{g} / \mathrm{mL})$ & Recuperação $(\%)^{*}$ \\
\hline Fosfato de betametasona & 60 & $99,95 \pm 0,22$ \\
& 80 & $100,18 \pm 0,14$ \\
Dipropionato de betametasona & 100 & $99,80 \pm 0,37$ \\
& 150 & $98,99 \pm 0,35$ \\
& 200 & $99,09 \pm 0,07$ \\
& 250 & $99,02 \pm 0,35$ \\
\hline
\end{tabular}

*Média \pm DPR, $n=3$.

loto. Os valores de resolução foram superiores a 2, indicando separação adequada dos picos, e os valores de assimetria ficaram próximos de 1 , 
Tabela 5. Resultados obtidos utilizando parâmetros modificados para demonstrar a robustez, teores dos fármacos na suspensão injetável, tempo de retenção, assimetria e resolução dos picos dos fármacos

\begin{tabular}{|c|c|c|c|c|c|c|c|c|}
\hline \multirow{2}{*}{ Parâmetro } & & \multicolumn{3}{|c|}{ Fosfato sódico de betametasona } & \multicolumn{3}{|c|}{ Dipropionato de betametasona } & \multirow{2}{*}{$\mathrm{R}_{\mathrm{s}}$} \\
\hline & & $\mathrm{T}(\%)$ & $t_{\mathrm{R}}$ & A & $\mathrm{T}(\%)$ & $t_{\mathrm{R}}$ & A & \\
\hline \multirow[t]{9}{*}{ Tampão pH } & 4,01 & $100,43^{1}$ & 6,95 & 1,11 & $118,44^{1}$ & 12,92 & 1,17 & 19,12 \\
\hline & & $99,72^{2}$ & 6,96 & 1,10 & $118,12^{2}$ & 12,92 & 1,18 & 19,15 \\
\hline & & $100,00^{3}$ & 6,96 & 1,10 & $117,54^{3}$ & 12,92 & 1,17 & 19,18 \\
\hline & 4,45 & 99,63 & 6,95 & 1,09 & 119,50 & 12,94 & 1,04 & 19,54 \\
\hline & & 99,55 & 6,95 & 1,08 & 119,02 & 12,94 & 1,05 & 19,48 \\
\hline & & 99,94 & 6,94 & 1,09 & 119,24 & 12,95 & 1,06 & 19,52 \\
\hline & 4,97 & 100,04 & 6,85 & 1,10 & 117,93 & 12,95 & 1,06 & 19,98 \\
\hline & & 99,51 & 6,84 & 1,10 & 118,00 & 12,94 & 1,06 & 20,01 \\
\hline & & 99,86 & 6,85 & 1,10 & 118,62 & 12,94 & 1,06 & 19,94 \\
\hline \multirow[t]{3}{*}{ Fluxo } & $1,5 \mathrm{~mL} / \mathrm{min}$ & 100,16 & 7,38 & 1,09 & 120,18 & 13,40 & 1,03 & 18,99 \\
\hline & & 100,21 & 7,38 & 1,09 & 120,19 & 13,40 & 1,03 & 18,96 \\
\hline & & 100,10 & 7,38 & 1,09 & 119,94 & 13,41 & 1,03 & 18,98 \\
\hline \multirow[t]{3}{*}{ Temperatura } & $30{ }^{\circ} \mathrm{C}$ & 100,54 & 5,98 & 1,08 & 121,85 & 12,66 & 1,02 & 24,82 \\
\hline & & 99,88 & 5,98 & 1,08 & 119,87 & 12,66 & 1,04 & 24,84 \\
\hline & & 100,25 & 5,97 & 1,09 & 119,76 & 12,65 & 1,03 & 24,81 \\
\hline \multirow[t]{5}{*}{ HPLC } & Shimadzu 10A & 100,03 & 8,78 & 1,07 & 120,52 & 14,94 & 1,01 & 15,53 \\
\hline & & 97,12 & 8,81 & 1,07 & 117,91 & 14,95 & 1,00 & 15,61 \\
\hline & & 99,77 & 8,75 & 1,06 & 121,32 & 14,91 & 1,00 & 15,93 \\
\hline & Média & 99,82 & & & 119,33 & & & \\
\hline & $\operatorname{DPR}(\%)$ & 0,73 & & & 1,03 & & & \\
\hline
\end{tabular}

1,2,3 - número de amostras; T(\%) - Teor dos fármacos na amostra; $\mathrm{t}_{\mathrm{R}}$ - Tempo de retenção (min); A - Assimetria; $\mathrm{R}_{\mathrm{s}}-\mathrm{R}_{\mathrm{s}}$ esolução; DPR - Desvio Padrão Relativo.

Tabela 6. Doseamento de diferentes marcas da suspensão disponíveis no mercado e parâmetros cromatográficos dos picos dos fármacos

\begin{tabular}{|c|c|c|c|c|c|c|c|}
\hline \multirow{2}{*}{ Produto } & \multicolumn{3}{|c|}{ Fosfato sódico de betametasona } & \multicolumn{3}{|c|}{ Dipropionato de betametasona } & \multirow{2}{*}{$\mathrm{R}_{\mathrm{s}}$} \\
\hline & Teor (mg/ampola) & $\mathrm{t}_{\mathrm{R}}$ & A & Teor (mg/ampola) & $t_{R}$ & A & \\
\hline A & 2,11 & 6,93 & 1,09 & 4,38 & 12,95 & 1,02 & 19,75 \\
\hline $\mathrm{B}$ & 2,04 & 6,92 & 1,09 & 5,28 & 12,93 & 1,01 & 19,74 \\
\hline $\mathrm{C}$ & 2,18 & 6,92 & 1,10 & 5,41 & 12,94 & 1,02 & 19,76 \\
\hline $\mathrm{D}$ & 2,45 & 6,92 & 1,10 & 6,20 & 12,96 & 1,02 & 19,80 \\
\hline $\mathrm{E}$ & 2,31 & 6,93 & 1,10 & 5,26 & 12,96 & 1,01 & 19,79 \\
\hline $\mathrm{F}$ & 2,12 & 6,93 & 1,09 & 5,44 & 12,97 & 1,01 & 19,81 \\
\hline $\mathrm{G}$ & 1,89 & 6,93 & 1,09 & 5,32 & 12,97 & 1,01 & 19,82 \\
\hline $\mathrm{H}$ & 2,46 & 6,93 & 1,11 & 7,14 & 12,97 & 1,02 & 19,74 \\
\hline I & 2,01 & 6,94 & 1,09 & 6,19 & 12,97 & 1,02 & 19,76 \\
\hline $\mathrm{J}$ & 2,44 & 6,93 & 1,12 & 6,41 & 12,97 & 1,02 & 19,70 \\
\hline
\end{tabular}

$\mathrm{t}_{\mathrm{R}}-$ Tempo de retenção (min); $\mathrm{A}-$ Assimetria; $\mathrm{R}_{\mathrm{s}}-$ Resolução.

indicando simetria dos picos nas diferentes condições. Desta forma, o método foi considerado robusto para análise do teor dos fármacos.

\section{Doseamento da suspensão injetável de DB e FB}

A fim de verificar a adequabilidade do método desenvolvido para as diferentes marcas comerciais de suspensões injetáveis, realizou-se o doseamento dos fármacos em dez produtos disponíveis no mercado brasileiro (A a J). Os valores de teor dos fármacos são apresentados na Tabela 6. Observa-se que os valores foram bastante variados. Porém, esta análise foi realizada utilizando-se o conteúdo inteiro das ampolas testadas, sem levar em conta o volume médio de cada lote. Segundo a Farmacopeia Brasileira, para líquidos injetáveis contendo $1 \mathrm{~mL}$, é preconizado um excesso mínimo de volume de $0,10 \mathrm{~mL}$ para líquidos móveis e de $0,15 \mathrm{~mL}$ para viscosos. ${ }^{5}$ Desta forma, os valores apresentados não devem ser interpretados como valores de uniformidade de dose.

Pode ser observado na Tabela 6 que o tempo de retenção se manteve 6,9 min para o FB e 12,9 min para o DB em todas as marcas comerciais testadas. A assimetria do pico variou de 1,09 a 1,11 para o FB e de 1,01 a 1,02 para o $\mathrm{DB}$, a resolução entre os picos dos fármacos variou de 19,74 a 19,82 e a pureza de pico (ratiogram) 
foi superior a 0,99 em todas as amostras para os dois fármacos. Os parâmetros cromatográficos demonstram que o método empregado na análise do teor foi eficiente para a separação e quantificação dos fármacos, mesmo na presença de diferentes constituintes das formulações farmacêuticas comerciais.

\section{CONCLUSÃO}

O método analítico empregando cromatografia líquida de alta eficiência em fase reversa e modo gradiente permitiu a detecção e quantificação simultânea dos fármacos FB e DB. A validação do método demonstrou a sua seletividade, linearidade, precisão, exatidão e robustez. Podendo ser considerado uma ferramenta eficiente para o controle de qualidade das suspensões injetáveis desta associação de fármacos, uma vez que não há método oficial para avaliar o teor destes fármacos nesta forma farmacêutica.

\section{REFERÊNCIAS}

1. Schellack, G.; Farmacologia: Uma abordagem didática, $1^{\mathrm{a}}$ ed., Fundamento: São Paulo, 2006.

2. Parfitt, K.; Martindale: The complete drug reference, $32^{\text {th }}$ ed., Pharmaceutical Press: Londres, 1999.

3. Korolkovas, A.; Burckhalter, J. H.; Química Farmacêutica, $1^{\mathrm{a}}$ ed., Guanabara Koogan: Rio de Janeiro, 1988.

4. Moffat, A. C.; Osselton, M. D.; Widdop, B.; Clarke's Analysis of Drugs and Poisons. $3^{\text {rd }}$ ed., Pharmaceutical Press: Londres, 2004, vol. 2.

5. Farmacopéia Brasileira, $5^{\mathrm{a}}$ ed., Fiocruz: Brasília, 2010.

6. Gil, E. S.; Controle Físico-Químico de Qualidade de Medicamentos, $2^{\mathrm{a}}$ ed., Pharmabooks: São Paulo, 2007.

7. Agência Nacional de Vigilância Sanitária - ANVISA; RE nº 899 de 29/05/2003: Guia para validação de métodos analíticos e bioanalíticos, Ministério da Saúde: Brasil, 2003, publicada em 02/06/2003.

8. ICH - International Conference on Harmonization of Technical Requeriments for registration of Pharmaceutical for Human use; $Q 2 B$ validation of Analytical procedure: methodology, 1996.
9. Agência Nacional de Vigilância Sanitária - ANVISA; RDC nº 37 de 6 de julho de 2009, publicada em 08/07/2009.

10. The United States Pharmacopeia, $33^{\text {th }}$ ed., United States Pharmacopoeial Convention: Rockville, 2010.

11. British Pharmacopoeia, The Stationery Office: London, 2011.

12. Farmacopea de los Estados Unidos Mexicanos, 5a ed., Secretaría de Salud: México, D.F., 1988.

13. Pharmacopée Française, $10^{\text {th }}$ ed., Ministère de la Santé: Paris, 2010.

14. European Pharmacopoeia, $3^{\text {th }}$ ed., Council of Europe: Strasbourg, 1997.

15. Farmacopeia Portuguesa, $8^{\text {a }}$ ed., Infarmed: Lisboa, 2005.

16. The International Pharmacopoeia, $4^{\text {th }}$ ed., World Health Organization: Geneva, 2008.

17. The Japanese Pharmacopoeia, $15^{\text {th }}$ ed., http://jpdb.nihs.go.jp/jp15e/JP15. pdf, acessada em Março 2011.

18. Wei, J.; Wang, J.; Chin. Pharm. J. 2002, 1.

19. Kedor-Hackmann E. R.; Gianotto, E. A.; Santoro, M. I.; Drug Dev. Ind. Pharm. 1998, 24, 553.

20. Samtani, M. N.; Schwab, M.; Nathanielsz, P. W.; Jusko, W. J.; J. Pharm. Sci. 2004, 93, 726.

21. Shou, M.; Galinada, W. A.; Wei, Y. C.; Tang, Q.; Markovich, R. J.; Rustum A. M.; J. Pharm. Biomed. Anal. 2009, 50, 356.

22. Lu, J.; Wei, Y.; Rustum, A. M.; J. Chromatogr., A 2010, 1217, 6932.

23. Fu, Q.; Shou, M.; Chien, D.; Markovich, R.; Rustum, A. M.; J. Pharm. Biomed. Anal. 2010, 51, 617.

24. Johnston, S. E.; Gill, N. L.; Wei, Y. C.; Markovich, R.; Rustum, A. M.; J. Chromatogr. Sci. 2010, 48, 733.

25. Bhosale, S. D.; Rajput, S. J.; J. AOAC Int. 2011, 94, 106.

26. Gika, H. G.; Papadoyannis, I. N.; Encyclopedia of Chromatography, $3^{\text {rd }}$ ed., Taylor \& Francis: Florida, Outubro, 2009.

27. Barros Neto, B.; Scarminio, I. S.; Bruns, R. E.; Como Fazer Experimentos: Pesquisa e desenvolvimento na ciência e na indústria, $2^{\mathrm{a}}$ ed., Ed. Da Unicamp: Campinas, 2003. 